\title{
Effect of Type II diabetic peripheral neuropathy on gait termination in the elderly
}

\author{
M.-R. Meier ${ }^{1}$, J. Desrosiers ${ }^{2}$, P. Bourassa ${ }^{2}$, J. Blaszczyk ${ }^{3}$ \\ ${ }^{1}$ Research Centre in Gerontology and Geriatrics, Sherbrooke Geriatrics University-Institute, University of Sherbrooke, Quebec, \\ Canada \\ ${ }^{2}$ Engineering Department, University of Sherbrooke, Sherbrooke, Quebec, Canada \\ ${ }^{3}$ Department of Neurophysiology, Nencki Institute of Experimental Biology, Warsaw, Poland
}

\section{Abstract}

Aims/hypothesis. Healthy elderly people can have difficulties in precisely terminating gait due to age-related decline. Diabetes mellitus accelerates the neurodegenerative process, which results in an additional decline in motor control. This biomechanical study investigated goal-oriented gait termination in healthy elderly and elderly diabetic subjects. The trajectories of the centre of pressure and the centre of mass during the gait termination process were analysed in particular. It was hypothesised that the pathology results in an unstable gait termination, expressed in larger overshoots of the centre of pressure and the centre of mass than in healthy control subjects.

Methods. A total of 15 subjects with Type II (non-insulin-dependent) diabetes mellitus with impaired foot sensitivity due to polyneuropathy (median, 66 years) were matched according to age, gender and body mass index with 15 healthy elderly subjects (median, 67 years). The participants walked at their own pace along the walkway and stopped in front of a marked stopping line while kinetic and kinematic data were recorded.

Results. The diabetic subjects approached the stopping line more slowly $(p=0.002)$ than the healthy elderly subjects. They also exhibited a weaker maximal braking force $(p=0.011)$ and a prolonged relative time to develop this force $(p=0.023)$. Despite this slower motion, the centre of pressure overshoots were larger in the diabetic subjects than in the healthy elderly $(p=0.027)$.

Conclusion/interpretation. The results show differences between healthy elderly and diabetic subjects during easy goal-oriented stopping tasks. Changes in gait termination parameters and the increased overshoots in particular document the pathology-related decline in postural stability. [Diabetologia (2001) 44: 585-592]

Keywords Gait termination, elderly, diabetes.
Received: 24 May 2000 and in revised form: 7 December 2000

Corresponding author: M.-R. Meier, PhD, Northwestern University Medical School, Prosthetic Research Laboratory and Rehabilitation Engineering Research Program, 345 East Superior Street, Room 144, Chicago, Illinois, 60611-4496, USA Abbreviations: A/P, anterior/posterior; M/L, medio/lateral; $\mathrm{COP}$, centre of pressure; COM, centre of mass; $\mathrm{HC}$, heel contact the onset of the stance phase in the stride cycle; TO, toe off: the onset of the swing phase; VST, Vibration Sensitivity Threshold; $\mathrm{F}_{1}$, maximal braking force; $\mathrm{F}_{2}$, maximal propelling force; $\mathrm{t}_{1}$, relative braking time; $\mathrm{t}_{2}$, relative time taken to develop the maximal braking force $\mathrm{F}_{1} ; \mathrm{t}_{3}$, relative time taken from the change of braking forces until TO; $\mathrm{t}_{4}$, relative time to develop the maximal propelling force $\mathrm{F}_{2} ; \mathrm{F}_{3}$, maximal stopping force; $t_{5}$, relative time until $\mathrm{HC} 3$ occurs; $\mathrm{t}_{6}$, relative time taken to develop the maximal stopping force $\mathrm{F}_{3} ; \mathrm{t}_{7}$, relative time taken until gait terminates biomechanically.
Human locomotion is well documented, including gait changes due to different pathologies and normal ageing [1-3]. Most of the published literature focuses, however, on steady-state locomotion: The participants are analysed while walking at a normal rhythm. But locomotion is not limited to steady-state body transportation. It needs to be adapted to a permanently changing environment and therefore requires an efficient control of postural stability. Velocity changes and stopping are examples of such adaptive processes. Despite the fact that daily activities require the ability to stop, only a handful of studies have investigated gait termination processes [4-6]. Terminating gait puts special demands on postural control, as a transition occurs from a dynamic situation (walking) to a quasi-static situation (standing). 
During the transition period, the central nervous system must control the development of appropriate braking forces, their directions and magnitude, so that a precise positioning of the centre of mass (COM) within the base of support is achieved. Hence, in order to terminate gait successfully a precise integration and co-ordination of sensory information, visual inputs and muscle activity is necessary. Normal physiological ageing results in a slowing down of neuronal processing and transmission of information, combined with decreased sensory information due to neurone loss [7-10]. Thus, situations in daily life that demand quick adaptation become more difficult with increasing age $[11,12]$.

Furthermore, pathologies are much more likely to occur with increasing age. Pathologies, such as arthritis and diabetes, can cause gait disorders $[13,14]$. It is therefore reasonable to assume that they also influence gait termination. Diabetes is one of the most common chronic diseases, with angiopathy, retinopathy, nephropathy and neuropathy as its main consequences [15-18]. The influences of peripheral neuropathy on gait and posture have been the topic of many studies $[13,19,20]$. They all agree that diabetic persons affected with peripheral neuropathy are more impaired than non-affected diabetic control subjects matched for age or persons without diabetes matched for age. Research on postural control (standing) shows that persons affected with diabetes and peripheral neuropathy show an increased anterior/posterior $(\mathrm{A} / \mathrm{P})$ and medio/lateral $(\mathrm{M} / \mathrm{L})$ centre of pressure (COP) range [13, 21]. Increased postural sway is usually a sign of greater instability $[9,10]$, further supported by the high risk factor of fall among independent elderly affected by peripheral neuropathy $[22,23]$. Other studies document that affected persons show a prolonged reaction time, slower walking velocity and a shorter stride length [19]. They are also 15 times more likely to report injuries during walking when compared to a non-neuropathic control subjects and hence feel significantly less safe in unusual conditions [24].

Other investigators were among the first to analyse rapid stopping behaviour and sudden turns in healthy elderly people [25-27]. They showed that in ninety-nine percent of cases, the elderly subjects fail to turn suddenly because of an inability to control the forward momentum in the available time [25]. Elderly people need significantly more time to execute a sudden stop than young people [26]. This can be attributed to the lengthened first phase of their responses and resulted in a higher post-cued acceleration. But a higher post-cue acceleration is not desirable for a sudden stop. Bearing in mind the fixed distance, it would either take more time to decelerate the whole body or require a larger force. Only elderly men were capable of partly compensating for this higher acceleration by developing a higher decelera- tion in the initial part of the deceleration phase [27]. This deceleration was also significantly higher when compared with young men. The authors point out that a possible reason for the gender differences found among the elderly participants could be due to the fact that elderly women are less able to develop ankle torque rapidly [28].

Little is known, however, about gait termination and its characteristics in the elderly population. Investigations of two key biomechanical parameters, the COP and centre of mass (COM), during goal-oriented gait termination in elderly people have not been reported so far. Furthermore, gait termination data for elderly subjects with diabetes and impaired foot sensitivity is lacking. Therefore, the purpose of the study was to quantify the biomechanical characteristics of goal-oriented gait termination and to establish the effect of diabetes. The COP and COM maximal overshoots, which occur during the gait termination process, have been analysed in particular. These overshoots are an indicator of co-ordination abilities and the efficiency of postural stabilization. The larger the overshoots during the gait termination process, the more difficult it was for the person to stop and maintain his or her equilibrium. It has been hypothesised that Type II diabetic persons with reduced foot sensitivity due to peripheral neuropathy exhibit larger COP and COM overshoots in $\mathrm{A} / \mathrm{P}$ and $\mathrm{M} / \mathrm{L}$ directions during stopping than healthy control subjects. Furthermore, the foot sensitivity and also the A/P COM velocity should correlate with the size of the COP and COM overshoots.

\section{Subjects and methods}

This project was approved by the Research Ethics Committee of the Sherbrooke Geriatric University-Institute. All participants gave their written consent.

Participants. Two groups (healthy and Type II diabetic elderly persons) were formed according to a non-probabilistic sampling design. The participants were recruited from the population of the city of Sherbrooke, Quebec, Canada, and the surrounding area. Each group was composed of 15 participants. Because of sex differences in gait, men and women were equally represented in each group according to the following eligibility criteria.

Participants were included if they were aged between 60 and 75 years and able to walk unassisted. The healthy elderly participants were matched according to age ( \pm 2 years), sex and body mass index (BMI) with the Type II diabetic persons. Care was taken not to violate the standard BMI classification of normal, over-weight and obese. The final acceptance was made on the result of measurements of fasting plasma glucose to ascertain whether they were free from diabetes. In order to exclude the possibility that the healthy elderly participants might have peripheral neuropathy due to causes other than diabetes, this group underwent a clinical examination using the scoring system previously developed [29]. This test is a global indicator of neurological status (sen- 
Table 1. Sociodemographic and anthropometric characteristics of the participants

\begin{tabular}{|c|c|c|c|}
\hline Group $^{a}$ & $\begin{array}{l}\text { Elderly } \\
(n=15)\end{array}$ & $\begin{array}{l}\text { Diabetic } \\
(n=15)\end{array}$ & $\begin{array}{l}p \\
\text { value }^{\mathrm{b}}\end{array}$ \\
\hline \multicolumn{4}{|l|}{ Continuous Variables } \\
\hline Age (years) & $67(2)^{\mathrm{c}}$ & $66(2)$ & 0.875 \\
\hline Height (m) & $1.63(0.005)$ & $1.63(0.005)$ & 0.712 \\
\hline Weight (kg) & $80.00(9.70)$ & $76.60(5.85)$ & 0.551 \\
\hline $\mathrm{BMI}\left(\mathrm{kg} / \mathrm{m}^{2}\right)$ & $30.65(3.37)$ & $30.17(3.24)$ & 0.826 \\
\hline $\begin{array}{l}\text { Vibration Sensitivity } \\
\text { Threshold (DfM) }\end{array}$ & $-0.97(1.42)$ & $2.90(0.66)$ & 0.001 \\
\hline \multicolumn{4}{|l|}{ Categorical Variables } \\
\hline \multicolumn{4}{|l|}{ Sex } \\
\hline Women & $7(46.7)^{\mathrm{e}}$ & $7(46.7)$ & 1.000 \\
\hline Men & $8(53.3)$ & $8(53.3)$ & \\
\hline \multicolumn{4}{|l|}{ Smoking Status } \\
\hline Non-smoker & $14(93.3)$ & $11(73.3)$ & 0.375 \\
\hline Smoker & $1(6.7)$ & $4(26.7)$ & \\
\hline \multicolumn{4}{|c|}{$\begin{array}{l}\text { Self-perceived activity } \\
\text { level }\end{array}$} \\
\hline 4. Very Active & $7(46.7)$ & $2(13.3)$ & 0.086 \\
\hline 3. Active & $5(33.3)$ & $6(40.0)$ & \\
\hline 2. Slightly Active & $3(20.0)$ & $4(26.7)$ & \\
\hline 1. Sedentary & 0 & $3(20.0)$ & \\
\hline \multicolumn{4}{|l|}{$\begin{array}{l}\text { Self-perceived health } \\
\text { status }\end{array}$} \\
\hline 4. Excellent & $7(46.7)$ & $1(6.7)$ & 0.003 \\
\hline 3. Good & $8(53.3)$ & $4(26.7)$ & \\
\hline 2. Fair & 0 & $6(40.0)$ & \\
\hline 1. Poor & 0 & $4(26.7)$ & \\
\hline \multicolumn{4}{|l|}{$\begin{array}{l}\text { Valk Graduation of } \\
\text { Neuropathy }^{\mathrm{d}}\end{array}$} \\
\hline 1. Not present & $11(73.3)$ & 0 & $<0.001$ \\
\hline 2. Mild & $4(26.7)$ & $8(53.3)$ & \\
\hline 3. Moderate & 0 & $4(26.7)$ & \\
\hline 4. Severe & 0 & $3(20.0)$ & \\
\hline
\end{tabular}

a Group abbreviations: Elderly = Healthy elderly participants; Diabetic $=$ Elderly Type II diabetic participants affected with peripheral neuropathy

${ }^{\mathrm{b}} p$ value associated with Wilcoxon Signed Ranks Test or chi squared

${ }^{c}$ Continuous variables: Median ( \pm semi-interquartile range)

${ }^{\mathrm{d}}$ Mean value of right and left leg, as no statistical difference was present between the two legs

${ }^{\mathrm{e}}$ Categorical variables: Frequency (percentage)

sory and motor) in the lower extremities. It includes, among other tests, pin-prick and light-touch sensation, sensory capacities of the ankle and the muscle strength of the gastrocnemius and extensor hallucis longus. The general scoring system is 0 point $=$ no abnormalities, 2 points $=$ impaired sensation in comparison with proximal sensation and 4 points = no sensation. In addition, the healthy elderly participants' vibration sensitivity threshold was also measured. The vibration sensitivity threshold (VST) was measured with the Vibrameter, Type IV (Somedic AB, Sweden). The VST was fixed according to the methods of limits (MOL) [30]. The threshold was detected by applying an increasing and decreasing vibration stimulus at the dorsal part of the big toe. The tested person identified this threshold as the minimal detectable amplitude of the applied vibration. The vibration was measured in microns and thereafter the age-corrected deviations from the mean (DfM) were calculated as indicated by the manufacturer. These age-corrected values were calculated using the re- gression equations based on the results of other investigators [31] who established normative values for the VST. The reported results are therefore adjusted with respect to the established values of this normative population and hence indicate the deviation from the population mean. This sets the results into a broader context.

The participants with Type II diabetes were included only if they had been diagnosed with peripheral neuropathy. The diagnosis of diabetic polyneuropathy was verified by clinical examination using the scoring system and described above [29]. In addition, a pronounced reduced vibration sensitivity had to be present. A person was considered as having this reduced vibration sensitivity when the measured value of at least one foot was more than two standard deviations above the mean of the normative population. Preference was given to non-invasive testing procedure and therefore electrodiagnostic tests for nerve function were not done.

The exclusion criteria were all factors that negatively influence gait and hence were also expected to negatively influence gait termination. Participants were excluded from the study if they had musculo-skeletal defects or any cardiac diseases (healthy elderly and diabetic persons), neurological defects (healthy elderly participants), neurological defects other than polyneuropathy (diabetic participants) or diabetes mellitus Type I or II (healthy elderly participants). The absence of diabetes in the healthy elderly group was tested by a fasting plasma glucose determination (FPG), with a normal range being between 3.8-6.1 mmol/1 [18]. Furthermore, participants were excluded if their visual acuity was below $6 / 21$ (metric) of the Snellen acuity chart, the threshold for low vision [32]. The sociodemographic and anthropometric characteristics of the participants are summarised in Table 1.

Definition of COP and COM maximal overshoots and foot placement. The COP and COM overshoots were measured once the participant reached a final stop. This was the case when both feet were stationary. Biomechanically, the end of gait termination is, however, defined as an A/P COM velocity lower than $0.05 \mathrm{~m} / \mathrm{s}$ [25]. The overshoots of both the COP and $\mathrm{COM}$ trajectories in $\mathrm{A} / \mathrm{P}$ and $\mathrm{M} / \mathrm{L}$ directions were measured with respect to the motion area centre (Fig.1). They were calculated with a customised programme, which allowed a visual identification of the overshoots. The motion area centre was also defined visually. It represents the centre of the motion area that the COP and the COM cover during quiet standing following gait termination. As the participants were asked to stand still for at least $15 \mathrm{~s}$ after they reached the final stop, this motion area was well defined.

The foot placement at gait termination defines the base of support. In our study, it was approximated as a rectangle. The width equals the maximal $\mathrm{M} / \mathrm{L}$ distance between the furthest lateral points of each foot, i.e. between the heads of the fifth metatarsals. The length equals the maximal A/P distance between the tip of the big toe and the contra-lateral heel. It is to be noted that the calculated base of support overestimates the effective base of support, as the participants were not required to place their feet in parallel at stop. Because no calculations of the COP and COM overshoots in relation with the base of support were done, this approach of presenting the base of support is adequate. The foot placement was calculated via a customised programme at HC3 (Fig. 2).

Data collection procedure. In a preliminary session, the prospective diabetic participants were tested for the presence of peripheral neuropathy by using the instruments and tests described above. For both groups, visual acuity, height and weight were measured before the gait termination data collection. 


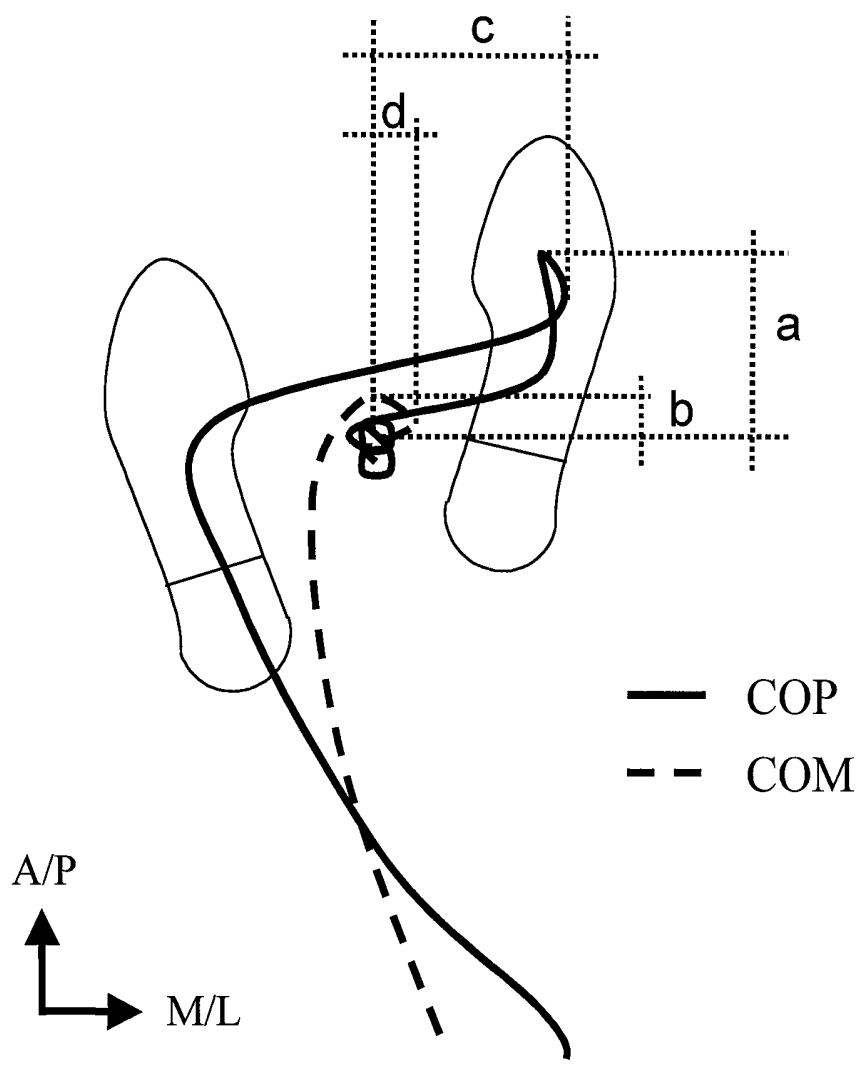

Fig.1. Definition of the COP and COM maximal overshoots. A/P direction: $\mathrm{a}=\mathrm{COP}$ overshoot $(\mathrm{cm}) ; \mathrm{b}=\mathrm{COM}$ overshoot $(\mathrm{cm}) ; \mathrm{M} / \mathrm{L}$ direction: $\mathrm{c}=\mathrm{COP}$ overshoot $(\mathrm{cm}) ; \mathrm{d}=\mathrm{COM}$ overshoot $(\mathrm{cm})$

In order to determine the COM location, ten infra-red markers were placed on the participant's body at specific anatomical points: big toes, medial malleoli, patellae, trochanter major (frontal plane position), acromii. In addition, eight digitised points, four on each foot, helped to identify the exact foot position at HC3. To avoid the hip markers being hidden by the arm-swing, the participants were asked to hook their thumbs into the belt used to attach the connector units of the system. A task familiarisation period preceded the experiment to ensure that none of the marker adhesives impeded the participants' movements. During this period, the optimal starting position was also determined to ascertain whether the participant's feet were on the force plates.

The experiment itself consisted of at least eight successful trials from whom five were chosen for further data analysis according to a standardised procedure. The participants were simply asked to walk at their preferred walking pace along an indicated walkway and to stop in front of the marked stopping line (Fig.2). They had roughly three meters to walk before one of their feet reached the first force platform. They were requested to stay immobile after full stop was reached until the sampling trial was finished. This allowed the motion area of the COP and the COM during quiet standing to be calculated.

Two camera towers of the OPTOTRAK-3020 optical system, Northern Digital, Waterloo, Canada, were used to register limb movements. Two force plates (OR6-5-1000, manufactured by AMTI, Watertown, Watertown, Mass., USA) recorded ground reaction forces during the stopping task. Both data collecting systems sampled at $60 \mathrm{~Hz}$.

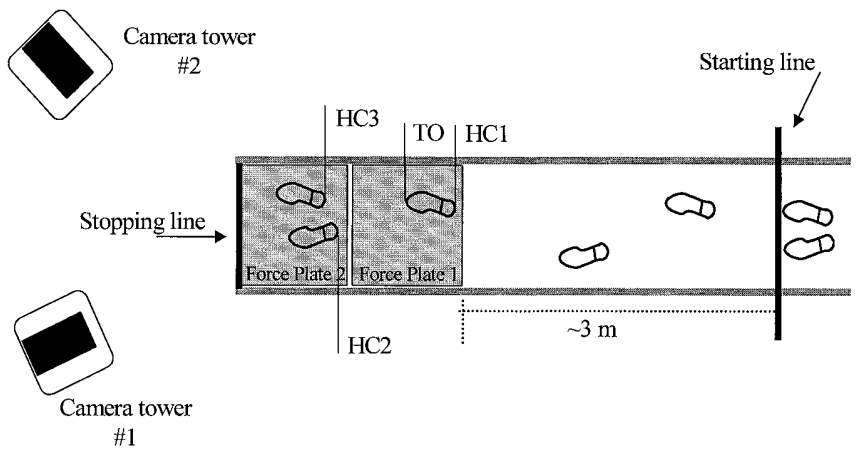

Fig. 2. Experimental set-up and categorisation of stopping procedure. Categorisation: HC1 until HC2: Approach Phase; $H C 2$ until $A / P C O M$ velocity $<0.05 \mathrm{~m} / \mathrm{s}$ : Stopping Phase. The Stopping Phase includes HC3, the event at which gait visually stops

Data and statistical analyses. The gait termination process was divided into two phases: the Approach and the Stopping Phases. The Approach Phase lasts from the first heel contact (HC1) to the second heel contact (HC2) (Fig. 2). The Stopping Phase begins with $\mathrm{HC} 2$ and ends when the A/P COM velocity was lower than $0.05 \mathrm{~m} / \mathrm{s}$, the definition of a full stop [25]. This phase includes the third heel contact (HC3), the event at which gait visually terminates.

These definitions are mainly based on the results of other investigators [6]. These authors divided the stopping time into three phases. Phase 1 was initiated by the left foot, which released the stopping signal and completed with the right heel contact. During this time a $10 \%$ reduction in gait velocity was achieved. Phase 2 was the main braking phase (right heel contact and second left heel contact) with a $73 \%$ reduction in gait velocity during this second step. Phase 3 included the fine-tuning of the COM by the COP. During Phase 3, the $\mathrm{COM}$ was brought into the final position, a position equivalent to standing still. Phase 1 of these investigations corresponds to the Approach Phase. The phases 2 and 3 proposed by these investigators have, however, been combined in this study under the heading of the Stopping Phase. This combination is the logical conclusion of the previous results. As mentioned above, the main braking occurred during phases 2 and 3 , hence these phases represent the Stopping Phase.

The A/P maximal forces, their timing and the A/P COM velocity were analysed during the entire gait termination process, i.e. the Approach and the Stopping Phase. Force plate data were time normalised so that $\mathrm{HC} 1$ to $\mathrm{TO}$ (toe off), which represents the stance phase of the Approach Phase, totalled $100 \%$. Furthermore, the Stopping Phase was normalised to $100 \%$ using a 3-point-rubber banding method (Matlab, The Math Works, Natick, Mass., USA).

Due to the non-parametric data distribution, the results are expressed as median and semi-interquartiles (continuous variables) or as frequency and percentage (categorical variables). Results of both groups were compared by either a Wilcoxon Signed Ranks test or by a chi-squared test. For the graphical representation of the results, the mean and the standard deviations are, plotted by convention. To establish the mutual relationship of the COP and COM overshoots, the $\mathrm{A} / \mathrm{P}$ velocity and the vibration sensitivity thresholds, the Spearman's correlation coefficient $\varrho$ was calculated. A $p$ value of less than 0.05 was accepted as being significant. All statistical analyses were carried out using the statistics software package SPSS for Windows (Standard Version 8.0 of SPSS Inc., Chicago, Ill., USA). 
Table 2. Results of A/P COM velocity, foot placement and $\mathrm{COP}$ and COM overshoots

\begin{tabular}{llll}
\hline Group $^{\text {a }}$ & $\begin{array}{l}\text { Elderly } \\
(n=15)\end{array}$ & $\begin{array}{l}\text { Diabetic } \\
(n=15)\end{array}$ & $\begin{array}{l}p \\
\text { value }^{\mathrm{b}}\end{array}$ \\
\hline A/P COM velocity (m/s) at & & & \\
HC1 & $1.13(0.18)^{\mathrm{c}}$ & $0.75(0.16)$ & 0.002 \\
HC2 & $0.80(0.14)$ & $0.61(0.11)$ & 0.015 \\
HC3 & $0.28(0.02)$ & $0.29(0.01)$ & 0.414 \\
Foot placement (cm) & & & \\
$\quad$ Width & $26.62(2.57)$ & $26.08(3.93)$ & 0.865 \\
Length & $29.88(1.68)$ & $29.49(2.05)$ & 0.865 \\
COP and COM maximal & & & \\
overshoots (cm) & & & \\
A/P COP Overshoot & $3.02(0.68)$ & $3.11(0.62)$ & 0.027 \\
M/L COP Overshoot & $2.55(0.64)$ & $3.14(1.45)$ & 0.029 \\
A/P COM Overshoot & $1.38(0.41)$ & $1.59(0.46)$ & 0.083 \\
M/L COM Overshoot & $1.49(0.39)$ & $1.69(0.60)$ & 0.256 \\
\hline
\end{tabular}

a Group abbreviations: Elderly = Healthy elderly participants; Diabetic $=$ Elderly Type II diabetic participants affected with peripheral neuropathy

${ }^{\mathrm{b}} p$ value associated with Wilcoxon Signed Ranks Test

${ }^{\mathrm{c}}$ Continuous variables: Median ( \pm semi-interquartile range)

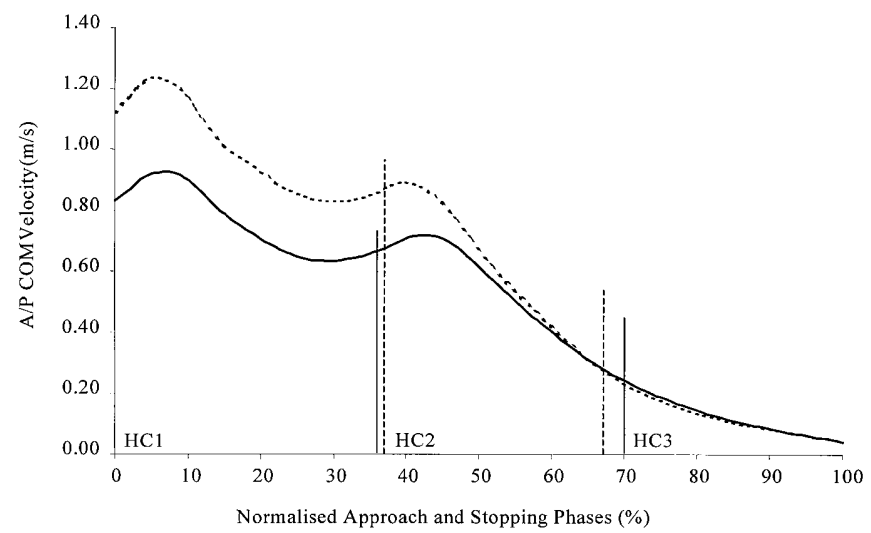

Fig.3. A/P COM velocity profile during the Approach and Stopping Phases. For each group, the whole stopping process ( $\mathrm{HC} 1$ to $\mathrm{A} / \mathrm{P}$ COM velocity $<0.05 \mathrm{~m} / \mathrm{s}$ ) has been set to $100 \%$. The corresponding resulting Approach and Stopping Phases are: Elderly: Approach Phase $(\mathrm{HC} 1$ to $\mathrm{HC} 2)=36 \%$; Stopping Phase $(\mathrm{HC} 2$ to $\mathrm{COM}<0.05 \mathrm{~m} / \mathrm{s})=64 \%(\cdots$ Elderly, mean $)$. Diabetic: Approach Phase (HC1 to $\mathrm{HC} 2)=37 \%$; Stopping Phase $(\mathrm{HC} 2$ to $\mathrm{COM}<0.05 \mathrm{~m} / \mathrm{s})=63 \%$ (-- Diabetic, mean)

\section{Results}

The participant sociodemographic characteristics (Table 1) show that both groups were similar in age, weight, height, BMI and also smoking status, although slightly more of the elderly Type II diabetic subjects with peripheral neuropathy smoked. The participants were also equal in their self-perceived activity level, but significantly different in self-perceived health status $(p=0.003)$. All elderly Type II diabetic subjects were affected by peripheral neuro-

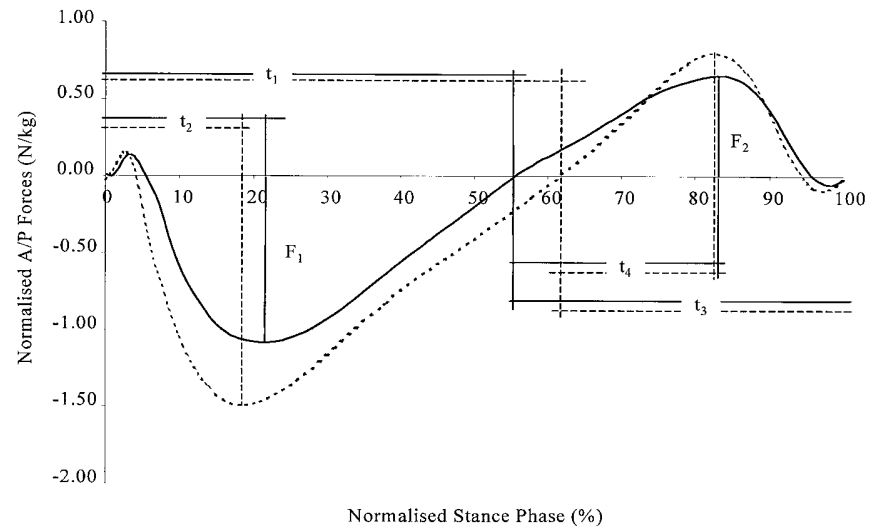

Fig. 4. A/P Forces of the Approach Phase (HC1 to HC2). Negative forces = braking forces; positive forces = propelling forces. Stance phase $=\mathrm{HC} 1$ to $\mathrm{TO}$ during the Approach Phase, normalised to a $100 \% ; F_{1}=$ maximal breaking force; $F_{2}=$ maximal propelling force; $t_{1}=$ relative time taken to change from braking to propelling forces; $t_{2}=$ relative time taken to develop the maximal braking force $\mathrm{F}_{1} ; \mathrm{t}_{3}=$ relative time taken from the change to the propelling forces until TO; $\mathrm{t}_{4}=$ relative time taken to develop the maximal propelling force $\mathrm{F}_{2}(\cdots$ Elderly, mean; -- Diabetic, mean)

pathy $(p<0.001)$ and also showed a significantly reduced vibration sensitivity threshold (VST) $(p=0.001)$. In contrast to this group, the VST of the healthy elderly subjects was excellent. Their general vibration sensitivity value was situated roughly 1 deviation below the mean of the normative population for this age group.

At the beginning of the Approach ( $\mathrm{HC1}$ ) and the Stopping Phases (HC2), the Diabetic A/P COM velocity of the elderly Type II diabetic subjects differed significantly from that of the healthy elderly subjects (Table 2, Fig.3). Only at HC3 were the differences in the A/P COM velocity of the two groups insignificant. Both groups placed their feet in a similar manner in order to terminate gait, resulting in an almost identical base of support $(p=0.865)$. The COP overshoot of the elderly Type II diabetic subjects in the A/P direction $(p=0.027)$ was, however, only statistically significantly larger than that in the healthy elderly subjects but had no clinical evidence. This contrasts with the COP overshoot in the M/L direction $(p=0.029)$ : The M/L COP overshoot was significantly (statistically and clinically) larger in the elderly Type II diabetic subjects than in the healthy elderly subjects (Table 2). There was no statistical difference in the $\mathrm{A} / \mathrm{P}$ and $\mathrm{M} / \mathrm{L}$ COM overshoots between the two groups.

The Approach Phase. The maximal braking force $\mathrm{F}_{1}$ of the healthy elderly subjects $(-1.59 \mathrm{~N} / \mathrm{kg} \pm 0.23)$ was larger than $\mathrm{F}_{1}$ of the elderly Type II diabetic subjects $(-0.98 \mathrm{~N} / \mathrm{kg} \pm 0.28)(p=0.011) \quad($ Fig. 4$)$. The maximal propelling force $\mathrm{F}_{2}$, however, was similar in both groups (Diabetic subjects $0.68 \mathrm{~N} / \mathrm{kg} \pm 0.09$, healthy subjects $0.76 \mathrm{~N} / \mathrm{kg} \pm 0.21 ; p=0.820)$. The transi- 


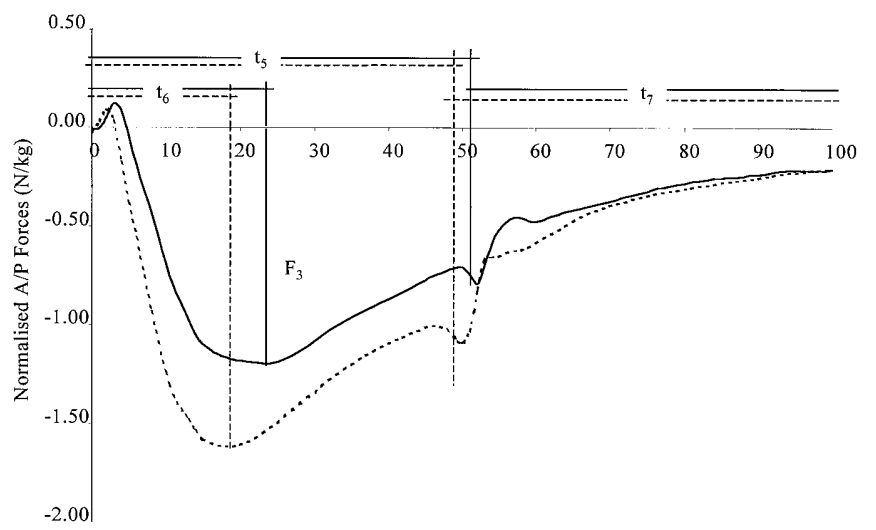

Normalised Stopping Phase (\%)

Fig. 5. A/P Forces during the Stopping Phase (HC2 until the $\mathrm{A} / \mathrm{P}$ COM velocity $<0.05 \mathrm{~m} / \mathrm{s}$ ). F3 = maximal stopping force; $\mathrm{t}_{5}=$ relative time taken until HC3 occurs, the event where gait visually terminates; $t_{6}=$ relative time taken to develop maximal stopping force; $t_{7}=$ relative time taken until gait terminates biomechanically, i.e. A/P COM velocity $<0.05 \mathrm{~m} / \mathrm{s}$ ( . . Elderly, mean; -- Diabetic, mean)

tion from braking to propulsion $\left(\mathrm{t}_{1}\right)$ occurred at $56 \% \pm 4.5$ in the elderly Type II diabetic subjects compared with $60 \% \pm 3.5$ in the healthy elderly subjects $(p=0.004)$. In contrast, the elderly Type II diabetic subjects needed longer to develop their maximal braking force $\left(\mathrm{t}_{2}\right)$ : occurrence of $\mathrm{F} 1$ at $22 \% \pm 3.5$ of the Approach Phase compared with $19 \% \pm 2$ in the healthy elderly subjects $(p=0.023)$. The maximal propelling force $\mathrm{F}_{2}$ peaked nearly at the same time: at $81 \% \pm 3.5$ of the entire Approach Phase for the elderly Type II diabetic subjects compared with $83 \% \pm 1.5$ for the healthy elderly subjects $(p=0.132)$.

The Stopping Phase. The maximal stopping force $\mathrm{F}_{3}$ of the healthy elderly subjects $(-1.56 \mathrm{~N} / \mathrm{kg} \pm 0.33)$ was again larger than that observed for the elderly Type II diabetic subjects $(-1.14 \quad \mathrm{~N} / \mathrm{kg} \pm 0.22)$ $(p=0.008)$ (Fig. 5). The results of the different relative times $\left(t_{5}, t_{6}\right.$ and $\left.t_{7}\right)$ contrast, however, with the results of the Approach Phase. There was no significant difference between the two groups. For the elderly Type II diabetic subjects, HC3 occurred at $52 \% \pm 4.5\left(t_{5}\right)$ of the Stopping Phase whereas HC3 of the healthy elderly subjects occurred at $50 \% \pm 4$ $(p=0.220)$. The maximal stopping force was reached in the elderly Type II diabetic subjects at $24 \% \pm 9$ of the Stopping Phase $\left(\mathrm{t}_{6}\right)$ versus $17 \% \pm 10$ in the healthy elderly subjects $(p=0.124)$.

In both groups, there are no correlations between the A/P COM velocities measured at $\mathrm{HC} 1, \mathrm{HC}$, $\mathrm{HC} 3$ and the COP and COM overshoots in both directions. In the healthy elderly subjects, the Spearman's correlation coefficient $\varrho$ ranged from 0.467 (A/P COM velocity at $\mathrm{HC1}$ and $\mathrm{A} / \mathrm{P} \mathrm{COP}$ overshoot; $p=0.079)$ to 0.168 (A/P COM velocity at $\mathrm{HC} 3$ and
M/L COM overshoot; $p=0.551$ ). In the elderly Type II diabetic subjects, the corresponding correlation coefficients were 0.109 (A/P COM velocity at $\mathrm{HC1}$ and $\mathrm{A} / \mathrm{P}$ COP overshoot; $p=0.669)$ to $0.484(\mathrm{~A} / \mathrm{P} \mathrm{COM}$ velocity at $\mathrm{HC} 3$ and $\mathrm{M} / \mathrm{L} \mathrm{COM}$ overshoot; $p=0.067)$. Furthermore, no correlation existed between the vibration sensitivity threshold (VST) and the COP and COM overshoots in both directions [ $\varrho$ ranges from 0.337 for VST and M/L COP overshoot $(p=0.069)$ to $\varrho=0.112$ for VST and M/L COM overshoot $(p=0.556)]$.

\section{Discussion}

Elderly people have difficulties in stopping suddenly, as the results of previous studies have shown [25-27]. Our study shows that these difficulties also occur in a goal-oriented daily stopping condition. It also shows that the existing difficulties of the healthy elderly people are exacerbated in the case of those with reduced vibration sensitivity. Diabetic subjects therefore show a reduced ability to terminate gait as shown by their larger $\mathrm{A} / \mathrm{P}$ and $\mathrm{M} / \mathrm{L}$ COP and COM overshoots.

The results of the A/P COM velocities confirm the general consensus that elderly diabetic people affected with peripheral neuropathy walk more slowly than their healthy counterparts matched for age [19]. This slowness could be observed even during the Approach Phase and, in part, during the Stopping Phase. Only from $\mathrm{HC} 3$ onwards, the event at which gait is visually terminated, is the corresponding A/P COM velocity nearly equal in both groups. The smaller maximal braking force $F_{1}$ and the longer relative time taken to develop this force $\left(t_{2}\right)$ indicates that the elderly Type II diabetic subjects do not seem to have initiated their stopping process during the Approach Phase as the elderly healthy people do. Two possible reasons for this situation could exist. First, because of their significantly slower walking velocity, elderly Type II diabetic subjects might not need to start their stopping procedure during this phase. It might be sufficient for them to stop their gait completely during the Stopping Phase itself. The altered locomotor strategy also suggests that elderly Type II diabetic subjects have adopted a 'slowness strategy'. This would allow them to reduce their disequilibrium so they might have more time to react. Second, the difference observed in the Approach Phase might be disease-induced as a possible result of the declined sensori-motor integration. Diabetic subjects affected with peripheral neuropathy show a prolonged reaction time $[19,33]$. The prolonged $t_{2}$ in the elderly Type II diabetic subjects would agree with the published literature. The same applies for $t_{6}$. It would not explain, however, the near similarity of $\left(\mathrm{t}_{1}+\mathrm{t}_{4}\right)$ in the elderly healthy and diabetic subjects. The rela- 
tive time taken to develop $F_{2}\left(t_{1}+t_{4}\right)$ might be the result of a different action. The braking period of the Approach Phase and the first part of the Stopping Phase (HC2 to HC3) are active periods in the stopping procedure. During these periods the main forces to terminate gait are produced. The two other periods, the propelling period of the Approach Phase and the period from $\mathrm{HC} 3$ to $\mathrm{A} / \mathrm{P} \mathrm{COM}$ of less than $0.005 \mathrm{~m} / \mathrm{s}$, are more passive periods without large actions. The period from HC3 to A/P COM of less than $0.005 \mathrm{~m} / \mathrm{s}$ includes the fine-tuning of the COM to a position equivalent to standing still [34]. The propelling period of the Approach Phase is noticeably reduced when compared with the propelling periods of the stance phases at normal walking speed. This also agrees with the results reported in the literature [5]. Reduced propelling forces during the Approach Phase reflect the roll to a stop, a fact, which applies to both groups. The characteristic prolonged reaction time in diabetic subjects might be more pronounced during active than during passive periods. This might explain why $t_{4}$ is not different between the elderly diabetic and healthy subjects.

The $\mathrm{A} / \mathrm{P}$ and $\mathrm{M} / \mathrm{L}$ COP overshoots are larger in the elderly diabetic subjects than in the healthy elderly subjects, demonstrating that the elderly diabetic subjects have difficulties in precisely co-ordinating their final stopping phase. Furthermore, the larger COP overshoots of the elderly diabetic subjects result in a larger motion area. Of note, the foot placement for both groups is very similar. Posture analyses have shown that the functional base of support diminishes with age $[35,36]$. In addition to the age-related reduced functional base of support, elderly diabetic subjects have to cope with diminished sensory information. As the quality of sensory information decreases, postural control is increasingly difficult and requires a wider margin of safety [10]. Taking these results into account, the demonstrated larger $\mathrm{A} / \mathrm{P}$ and $\mathrm{M} / \mathrm{L}$ COP overshoots reveal that the elderly diabetic subjects are closer to their stability limit than the healthy elderly subjects. In other words, they become less tolerant of unexpected environmental changes, which might occur during the transition period and hence would require quick adaptations.

The results of this study also show that the impact of peripheral neuropathy seems to be partly compensated for by walking velocity. Although neither of the correlations between the VST and the COP and $\mathrm{COM}$ overshoots in either direction reached the level of significance, the negative effect of peripheral neuropathy could be observed. This effect was, however, masked by mechanisms, which help to control the COP and COM overshoots. One of these mechanisms is the 'slowness strategy'. The elderly diabetic subjects walked with a significantly slower walking velocity compared with the elderly healthy subjects. Therefore, neuropathy as a whole has a negative ef- fect on the walking characteristics. It has to be remembered that reduced vibration sensitivity (VST) is only one feature of neuropathy. It could be that other characteristics such as reduced nerve conduction velocities, reduced muscle forces, especially plantar flexor muscles, or a combination of the different characteristics could have a larger effect on the overall stopping behaviour than VST alone. The results obtained therefore have to be interpreted with caution.

Unexpectedly, there is no relation between the $\mathrm{A} / \mathrm{P}$ COM velocity and the COP and COM overshoots. This could be explained by the fact that all participants were allowed to choose their preferred walking speed. Walking speed is influenced by external criteria, such as task and walking distance (in the present case, the relatively short distance that could have restricted the participants in fully developing their normal walking speed) and by internal criteria such as age and health status. These criteria might have lead to a walking speed that enables the participants to control their COP and COM trajectories as shown. Velocity controlled trials are, however, necessary to establish the lack of effect of A/P COM velocity on the COP and COM overshoots.

It has been assumed that the main stopping procedure occurs in the $\mathrm{A} / \mathrm{P}$ direction. Therefore, our study's ground reaction forces and COM velocity analyses were limited to this direction only. An additional limit of the study is the lack of muscle strength data for the plantar flexors. It has been shown that development of rapid ankle torques is impaired in elderly people and is further reduced in people with diabetes [28, 37]. In retrospect, another useful tool would have been electromyographic (EMG) recording. EMG data combined with actual muscle strength might have helped to interpret the COP and COM overshoot differences shown.

In conclusion, the study documents that differences between elderly Type II diabetic people affected with peripheral neuropathy and healthy elderly people also exist in easy goal-oriented every-day stopping conditions. The differences are expressed in a slower A/P COM velocity during the entire Approach Phase and during most subcomponents of the Stopping Phase. Despite the slower walking velocity, the $\mathrm{A} / \mathrm{P}$ and $\mathrm{M} / \mathrm{L}$ COP overshoots of the elderly Type II diabetic subjects are larger than in the elderly healthy subjects. Therefore, the co-ordination abilities of elderly Type II diabetic subjects are impaired, leading to reduced stability during the critical transition from the dynamic to the quasi-static condition. They are therefore at a higher risk of falling.

In the future, gait termination tests might be used as an easy clinical test to establish dynamical instability problems. Almost every pathology, including diabetes, impairs postural stability. These instabilities are not always obvious during steady state, unre- 
strained locomotion. Gait termination, as a transient phenomenon, is able to exhibit the underlying impairments. It might therefore have the potential to be used as a clinical testing instrument.

Acknowledgements. The study was partly supported by the National Science and Engineering Research Council of Canada (NSERC) and the Medical Research Council of Canada (CRM). Their contribution is greatly appreciated.

\section{References}

1. Perry J (1992). Gait analysis. Slack Inc., Thorofare, USA

2. Sudarsky L (1990). Geriatrics: gait disorders in the elderly. NEJM 322(20): 1441-1446

3. Maki BE (1997). Gait changes in older adults: predictors of fall or indicators of fear? JAGS 45: 313-320

4. Yamashita T and Katoh R (1976). Moving pattern of point of application of vertical resultant force during level walking. J Biomech 9: 93-99

5. Hase K and Stein RB (1998). Analysis of rapid stopping during human walking. J Neurophysiol 80(1): 255-261

6. Jian Y, Winter DA, Ishac MG and Gilchrist L (1993). Trajectory of the body COG and COP during initiation and termination of gait. Gait Posture 1: 9-22

7. Maki BE and McIlroy WE (1996). Postural control in older adult. Clin Geriatr Med 12(4): 635-658

8. Welford AT (1984). Between bodily changes and performance: some possible reasons for slowing with age. Exp Aging Res 10(2): 73-88

9. Blaszczyk JW, Lowe DL and Hansen PD (1993). Postural sway and perception of the upright stance stability borders. Perception 22: 1333-1341

10. Blaszczyk JW, Lowe DL and Hansen PD (1994). Ranges of postural stability and their changes in the elderly. Gait Posture 2(1): 11-17

11. Prudham D and Grimley Evans J (1981). Factors associated with falls in the elderly: a community study. Age and Ageing 10: 141-146

12. Nevitt MC (1997). Falls in the elderly: risk factors and prevention. In: Masdeu JC, Sudarsky L and Wolfson L (eds.) Gait disorders of aging: falls and therapeutic strategies. Lippincott-Raven, Philadelphia, PA, pp 13-36

13. Cavanagh PR, Simoneau GG and Ulbrecht JS (1993). Ulceration, unsteadiness, and uncertainty: the biomechanical consequences of diabetes mellitus. J Biomech 26: S23-S40

14. Alexander NB (1996). Differential diagnosis of gait in older adults. Clin Geriatr Med 12(4): 689-703

15. WHO (1985). Diabetes mellitus. Report of a WHO study group. World Health Organisation Technical Report Series 727, World Health Organisation, Geneva

16. Seaquist ER (1998). Microvascular complications of diabetes: Strategies for managing retinopathy, nephropathy, and neuropathy. Postgrad Med 103(1): 61-63,66-68. (Published erratum in Postgrad Med 1998; 103(5): 38)

17. Sherwin RS (1996). Diabetes mellitus. In: Cecil Textbook of Medicine. Benneth JC and Plum F (eds.), WB Saunders Company

18. Meltzer S, Leiter L, Daneman D, Gerstein HC, Lau D, Ludwig S et al. (1998). 1998 clinical practice guidelines for the management of diabetes in Canada. CMAJ 159(8 Suppl): S1-S29

19. Courtemanche R, Teasdale N, Boucher P, Fleury M, Lajoie Y and Bard C (1996). Gait problems in diabetic neuropathic patients. Arch Phys Med Rehabil 77: 849-855
20. Lord SR, Caplan GA, Colagiuri R, Colagiuri S and Ward JA (1993). Sensori-motor function in older persons with diabetes. Diabet Med 10: 614-618

21. Katoulis EC, Ebdon-Parry M, Hollis S, Harrison AJ, Vileikyte L, Kulkarni J et al. (1997). Postural instability in diabetic neuropathic patients at risk of foot ulceration. Diabet Med 14: 296-300

22. Koski K, Luukinen H, Laippala P and Kivelä S-L (1998): Risk factors for major injurious falls among the homedwelling elderly by functional abilities. Gerontology 44 : 232-238

23. Richardson JK and Hurvitz A (1995). Peripheral Neuropathy: A true risk factor for falls. J Gerontol 50A (4): M211M215

24. Cavanagh PR, Derr JA, Ulbrecht JS, Moser RE and Orchard TJ (1992). Problems with gait and posture in neuropathic patients with insulin dependent diabetes mellitus. Diabet Med 9: 469-474

25. Cao C, Ashton-Miller JA, Schultz AB and Alexander NB (1997). Abilities to turn suddenly while walking: effects of age, gender and available response time. J Gerontol 52A(2): M88-M93

26. Cao C, Schultz AB, Ashton-Miller JA and Alexander NB (1998). Sudden turns and stops while walking: kinematic sources of age and gender differences. Gait Posture 7: $45-52$

27. Cao C, Ashton-Miller JA, Schultz AB and Alexander NB (1998). Effect of age, available response time and gender on ability to stop suddenly when walking. Gait Posture 8: 103-109

28. Thelen DG, Schultz AB, Alexander NB and Asthon-Miller JA (1996). Effects of age on rapid ankle torque development. J Gerontol Med Sci 51A: M226-M232

29. Valk GD, Nauta JJ, Stijers RLM and Bertlsman FW (1992). Clinical examination versus neurophysiological examination in the diagnosis of diabetic polyneuropathy. Diabet Med 9: 716-721

30. Gerr F, Hershman D and Letz R (1990). Vibrotactile threshold measurement for detecting neurotoxicity: reliability and determination of age-and height standardised normative values. Arch Environ Health 45(3): 148-154

31. Goldberg JM and Lindblom U (1979). Standardised method of determining vibratory perception thresholds for diagnosis and screening in neurological investigation. J Neurol Neuros Psychiatry 42: 793-803

32. Ministère des affaires sociales (1982). L'organisation du réseau de services de réadaptation aux personnes handicapées à cause d'une déficience visuelle: Plan et orientation. Québec, Gouvernement du Québec, Canada

33. Andersen H and Nogensen PH (1997). Disordered mobility of large joints in association with neuropathy in patients with long-standing insulin-dependent diabetes mellitus. Diabet Med 14(3): 221-227

34. Winter DA (1991). The biomechanics and motor control of human gait: normal, elderly and pathological. Second edition, Waterloo Biomechanics, Waterloo, Canada

35. King MB, Judge JO and Wolfson L (1994). Functional base of support decreases with age. J Gerontol Med Sci 49(6): M258-M263

36. Kozak K, Ashton-Miller JA and Nyquist L (1997). Effects of age and base of support on maximum forward reach. American Society of Biomechanics, 21. Annual Meeting, Clemson University, South Carolina, Sept. 24-27

37. Mueller MJ, Minor SC, Schaaf JA, Strube MJ and Sahrmann SA (1995). Relationship of plantar-flexor peak torque and dorsiflexion range of motion to kinetic variables during walking. Phys Ther 75: 1196-1200 